\section{Dor dentária e fatores associados em adolescentes brasileiros: a Pesquisa Nacional de Saúde do Escolar (PeNSE), Brasil, 2009}

\author{
Dental pain and associated factors in Brazilian \\ adolescents: the National School-Based Health \\ Survey (PeNSE), Brazil, 2009
}

\author{
${ }^{1}$ Faculdade de Odontologia, \\ Universidade Federal de \\ Goiás, Goiânia, Brasil. \\ 2 Secretaria de Vigilância em \\ Saúde, Ministério da Saúde, \\ Brasília, Brasil. \\ 3 Secretaria de Atenção à \\ Saúde, Ministério da Saúde, \\ Brasília, Brasil. \\ 4 Programa de Pós-graduação \\ em Saúde Coletiva, \\ Universidade Federal \\ de Santa Catarina, \\ Florianópolis, Brasil. \\ Correspondência \\ M. C. M. Freire \\ Faculdade de Odontologia \\ Universidade Federal de \\ Goiás. \\ Praça Universitária \\ Campus I, Goiânia, GO \\ 74605-220, Brasil. \\ mcmfreire@yahoo.com.br
}

\section{Abstract}

The aim of this study was to assess the prevalence of dental pain and associated socio-demographic and behavioral factors in Brazilian adolescents, using data from the National School-Based Health Survey (PeNSE), Brazil, 2009. The survey was conducted by the Brazilian Institute of Geography and Statistics (IBGE) and Ministry of Health in students 11 to 17 years of age or older in the 27 State capitals, using a self-administered questionnaire. Analyses included Poisson regression following a hierarchical approach. Prevalence of dental pain in the sample $(n=54,985)$ in the previous six months was $17.8 \%$ (95\%CI: 17.5-18.1). Higher prevalence was associated with female gender, age 14 years and over, racial self-identification as black, brown, or indigenous, enrollment in public schools, lower maternal schooling, not living with the mother, history of smoking or drinking, less frequent toothbrushing, and heavy consumption of sweets and soft drinks. Dental pain was thus associated with socio-demographic factors and health-related behaviors.

Toothache; Risk Factors; Socioeconomic Status; Oral Health
Maria do Carmo Matias Freire 1

Cláudio Rodrigues Leles 1

Luciana Monteiro Vasconcelos Sardinha 2

Moacir Paludetto Junior 3

Deborah Carvalho Malta 2

Marco A. Peres 4

\section{Introdução}

A dor de dente, ou odontalgia, é geralmente definida como um tipo de dor orofacial originada nos dentes e suas estruturas de suporte, como consequência de doenças como cárie, periodontais e traumatismo dentário ${ }^{1}$. Essa condição é motivo frequente de procura por serviços odontológicos, de automedicação e causa impacto significativo na vida dos indivíduos e da sociedade, constituindo assim, um importante problema de saúde pública 2,3,4. Nesse sentido, a redução da ocorrência da dor de origem dento-bucal e craniofacial constitui uma das metas globais para a saúde bucal no ano de 2020, propostas pela Organização Mundial da Saúde, Federação Dentária Internacional e Associação Internacional de Pesquisa Odontológica 5.

A partir da década de 1990, tem havido um crescente interesse pelo tema, resultando em estudos epidemiológicos que têm ampliado seu escopo e investigado não só a prevalência do problema, mas também os fatores individuais e contextuais a ele associados 6,7 e o seu impacto nas atividades diárias das pessoas 8,9. Dentre os fatores associados a esse agravo, destacam-se os demográficos, socioeconômicos, familiares, psicológicos e culturais, além da condição de saúde bucal e comportamentos relacionados 1,6,10. Com base na literatura publicada, Bastos et al. ${ }^{11}$ propuseram um modelo conceitual de determinação social da dor de dente, em que a ligação entre 
estrutura social e saúde bucal se dá por meio de vias materiais, comportamentais e psicossociais.

Grande parte dos estudos sobre esse tema tem sido realizada em crianças e adolescentes. Nessa fase da vida, a principal causa da dor de dente é a cárie dentária, e a dor pode afetar negativamente a alimentação, as brincadeiras e o sono 12,13 , bem como a concentração na escola 8,9. No Reino Unido, a dor de dente foi o principal problema relatado pelos pais de crianças e adolescentes no levantamento nacional de saúde bucal realizado em 2003 14. Um trabalho realizado nos Estados Unidos sugere que a dor de dente é um potencial indicador das desigualdades em saúde bucal na população infantil 15 .

No Brasil, de acordo com o levantamento nacional de saúde bucal de 2003 16,17, a prevalência da dor de dente nos seis meses anteriores ao estudo foi de $35,7 \%$ em indivíduos de 15 a 19 anos. Desde o início dos anos 2000, alguns trabalhos sobre o tema têm sido realizados em escolares de municípios das regiões Sul, Sudeste e Nordeste $7,8,13,18,19,20,21$. Nesses estudos, a prevalência de dor nos últimos meses em crianças e adolescentes variou de 15,6 a $36,6 \%$, dependendo da idade e do período recordatório.

O relato de dor de dente foi contemplado também no questionário da primeira Pesquisa Nacional de Saúde do Escolar (PeNSE), realizada pelo Ministério da Saúde e pelo Instituto Brasileiro de Geografia e Estatística (IBGE) em 2009, que teve como finalidade investigar diversos fatores de risco e proteção à saúde numa amostra representativa de escolares adolescentes brasileiros 22 . Buscou-se com essa pesquisa, informações confiáveis que permitam conhecer a magnitude dos fatores relacionados à saúde, orientar intervenções em saúde adequadas ao adolescente e avaliar o impacto das mesmas. A inclusão do componente saúde bucal em inquéritos nacionais de saúde com essas características é importante e pode contribuir para a construção de um modelo de vigilância em saúde mais integrado e efetivo. As informações sobre dor de dente no banco de dados da PeNSE possibilitam a análise não só da sua ocorrência na população estudada, mas também explorar a sua associação com fatores de risco e proteção à saúde geral.

O presente trabalho objetiva estimar a prevalência da dor de dente em adolescentes brasileiros e analisar seus fatores associados, utilizando os dados da PeNSE de 2009.

\section{Métodos}

No presente trabalho, foram usados os dados da primeira PeNSE, realizada por meio de estudo transversal de base escolar. A pesquisa foi coordenada pelo Ministério da Saúde e executada pelo IBGE. Contou ainda com o apoio institucional do Ministério da Educação e parcerias com instituições de ensino e pesquisa no Brasil. A metodologia e os resultados principais do estudo encontram-se publicados 22,23 , e os aspectos mais importantes serão descritos a seguir.

\section{População de estudo}

A população de estudo foi composta por alunos cursando, no ano de 2009, o nono ano do ensino fundamental de escolas públicas e privadas das 26 capitais de estados brasileiros e o Distrito Federal. A PeNSE foi realizada no ambiente escolar pela maior facilidade de acesso aos adolescentes, pois, segundo dados do IBGE, a grande maioria dos adolescentes entre 10 e 14 anos (97\%) encontrava-se matriculada em escolas em 200724. A amostra de alunos matriculados foi estimada com base no Censo Escolar 2007, realizado pelo Instituto Nacional de Estudos e Pesquisas Educacionais Anísio Teixeira, do Ministério da Educação 25. Esse público da pesquisa se justifica por ter o mínimo de escolarização necessário para responder ao questionário autoaplicável e por ser a série que concentra a faixa etária de 13 a 15 anos.

A amostragem foi probabilística, estratificada, por conglomerados e em dois estágios de seleção. No primeiro, foram selecionadas as escolas (Unidade Primária de Amostragem - UPA) e, destas, foram escolhidas as turmas (Unidade Secundária de Amostragem - USA) que tiveram todos os alunos presentes no dia da pesquisa incluídos na amostragem.

A estratificação das UPA foi segundo sua localização geográfica, com cada estrato sendo composto por uma capital de estado brasileiro ou o Distrito Federal, resultando em 27 estratos geográficos. O tamanho da amostra foi calculado em cada um dos estratos de modo a estimar prevalências de $50 \%$, com um erro absoluto da ordem de três pontos porcentuais, e nível de significância de cinco pontos porcentuais. A amostra de cada estrato foi alocada proporcionalmente ao número de escolas segundo dependência administrativa - pública e privada. Foram excluídas as escolas com menos de 15 alunos na série desejada e as turmas do turno noturno.

Foi sorteada aleatoriamente uma turma de nono ano para as escolas selecionadas que possuíam uma ou duas turmas desta série, e duas turmas naquelas com três ou mais. Em cada turma selecionada todos os estudantes presentes no dia da coleta de dados foram convidados a participar do inquérito. Não participaram os alunos 
faltantes que usualmente frequentavam as aulas, os alunos presentes que não quiseram responder ao questionário e aqueles que não preencheram a variável sexo do questionário.

Os pesos amostrais atribuídos a cada um dos estudantes na base de dados final foram construídos levando-se em conta todas essas perdas, portanto devem refletir a população-alvo, formada pelos alunos do nono ano do ensino fundamental que frequentavam regularmente as aulas nas escolas privadas e públicas das capitais estaduais e do Distrito Federal, nos turnos diurnos. Detalhes do procedimento amostral podem ser obtidos em outra publicação 22 .

\section{Coleta dos dados}

O instrumento foi um questionário estruturado e autoaplicável dividido em módulos por assunto, que incluíram as características sociodemográficas, alimentação, imagem corporal, atividade física, tabagismo, consumo de álcool e outras drogas, saúde bucal, comportamento sexual, violência, acidentes, segurança e experiências em casa e na escola. A coleta de dados foi realizada pelo microcomputador de mão Personal Digital Assistant (PDA).

O questionário foi construído com base em instrumentos utilizados no Brasil 26,27,28 e em outros países 29,30, e pré-testado em uma amostra de escolas. O trabalho de campo foi realizado por equipes estaduais do IBGE entre março e junho de 2009.

\section{Variáveis incluídas no presente estudo}

A variável dependente investigada foi o relato de dor de dente nos últimos seis meses antes da coleta dos dados, que foi obtida por meio da seguinte questão: "Nos últimos seis meses, você teve dor de dente? Excluir dor causada por uso de aparelho de dente". As categorias de resposta foram: Sim, Não e Não sei/não me lembro.

As variáveis independentes foram as características sociodemográficas (sexo, idade, cor/raça autorrelatada, condição socioeconômica aferida pela escolaridade materna, se o escolar residia com a mãe e o tipo de escola), hábitos e comportamentos relacionados à saúde (uso de cigarro, consumo de álcool e atividade física), comportamentos relacionados à cárie dentária (consumo de alimentos que contêm açúcar e frequência diária de escovação).

A idade foi categorizada em 11-13, 14-16 e 17 ou mais. Cor da pele/raça autorreferida foi categorizada em cinco grupos: branca, preta, parda, amarela e indígena. Para a variável mora com a mãe, as categorias foram sim e não. A escola- ridade materna foi coletada de acordo com os anos de estudos completos com sucesso e categorizada em $>11$ anos, $8-11$ anos $\mathrm{e}<8$ anos. $\mathrm{O}$ tipo de escola foi determinado pela dependência administrativa, se pública ou privada. Para uso de cigarro utilizou-se a questão: “Alguma vez na vida você já fumou cigarro, mesmo uma ou duas tragadas?", categorizada em sim e não. $\mathrm{O}$ consumo de álcool foi obtido por meio da questão: “Alguma vez na vida, você já experimentou bebida alcoólica?”, categorizadas em sim e não. A atividade física foi baseada no total de horas de atividades acumuladas, somando-se os tempos gastos com estas atividades nos últimos sete dias, obtendo-se as seguintes categorias: inativo; insuficientemente ativo (subdividido entre os que praticaram atividade física de 1 a 149 minutos e os que praticaram atividade física de 150 a 299 minutos); e ativo (praticaram 300 minutos ou mais de atividade física). O consumo de alimentos que contêm açúcar, como guloseimas e refrigerantes, foi obtido por meio de questões sobre a frequência semanal, categorizado em baixo consumo (nenhum a quatro dias na semana) e alto consumo (pelo menos cinco dias na semana). A frequência diária de escovação foi categorizada em três ou mais vezes, duas vezes, e uma ou nenhuma.

\section{Análise dos dados}

A análise dos fatores associados à prevalência da dor de dente foi realizada levando-se em consideração um modelo hierárquico para determinação do agravo (Figura 1) 7,31. Foram incluídas variáveis retiradas do questionário PeNSE, divididas em três blocos. No nível mais distal situamse as características demográficas (bloco 1) como sexo, idade e cor/raça que hipoteticamente influenciam as condições socioeconômicas (bloco 2), que por sua vez determinam comportamentos relacionados à saúde geral e saúde bucal (bloco 3), nível mais proximal do desfecho avaliado.

A análise dos dados incluiu a distribuição das frequências das categorias das variáveis e cálculo das prevalências da dor de dente (porcentagem e intervalo de 95\% de confiança - IC95\%). Para identificação das variáveis associadas à dor de dente foi utilizada a razão de prevalência como medida de efeito com IC95\%, obtida pela análise de regressão de Poisson. Todas as variáveis com significância menor que 0,20 na análise bivariada foram incluídas no modelo multivariado. A análise de regressão de Poisson múltipla foi realizada de acordo com o modelo hierarquizado estruturado nos blocos de variáveis descritos anteriormente. Partiu-se de um modelo que incluiu todas as variáveis do mesmo bloco, sendo 
Modelo hierárquico proposto para determinação da dor de dente em escolares das capitais brasileiras. Pesquisa Nacional de Saúde do Escolar (PeNSE), Brasil, 2009.

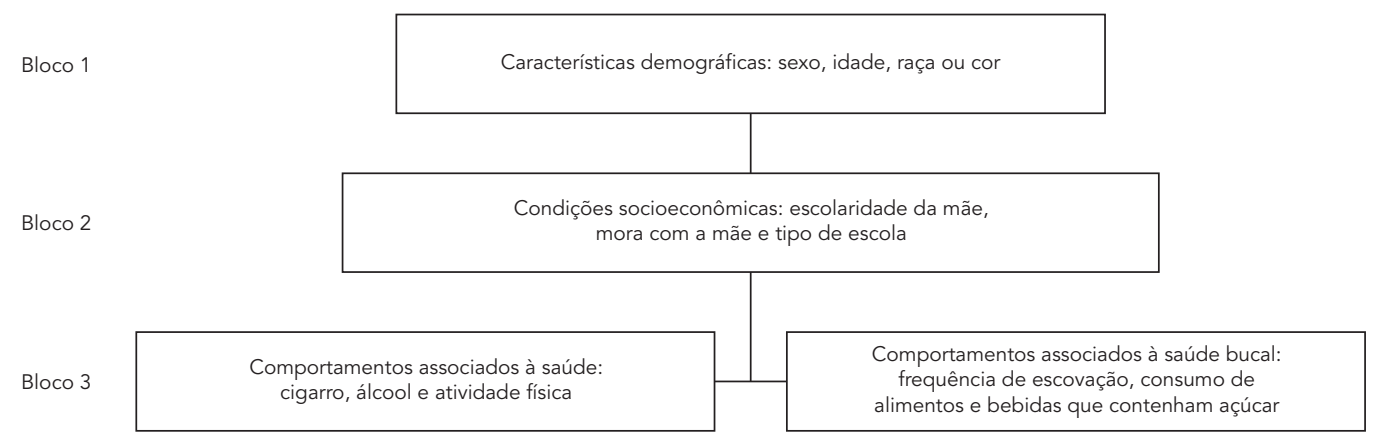

retiradas, uma a uma, as variáveis com valor de p maior que 0,05. Obtiveram-se três modelos de regressão, nos quais as razões de prevalência foram ajustadas pelas variáveis do mesmo nível ou superiores. Em todas as análises considerou-se o efeito do desenho amostral para amostras complexas, utilizando-se pesos amostrais calculados no módulo Complex Samples do programa estatístico SPSS 17.0 (SPSS Inc., Chicago, Estados Unidos). Em todas as análises descritivas e inferenciais utilizou-se o programa Stata 9.1 (Stata Corp., College Station, Estados Unidos).

\section{Aspectos éticos}

A PeNSE foi aprovada pelo Comitê Nacional de Ética em Pesquisa do Ministério da Saúde, sob o parecer $n^{\circ}$. 11.537. A realização do estudo foi precedida do contato com as secretarias estadual e municipal de Educação e Saúde e com a direção das escolas selecionadas em cada município. A participação dos alunos foi voluntária. O questionário poderia ser respondido em sua totalidade ou em parte. Todas as informações, tanto do aluno quanto da escola, foram confidenciais e não identificadas.

\section{Resultados}

Inicialmente, foram selecionadas 1.507 escolas, porém, por motivos operacionais foram pesquisadas 1.453 , resultando em perda de $3,6 \%$ de escolas. Foram pesquisadas 2.175 turmas, correspondendo, segundo informações das próprias escolas, a 72.872 alunos matriculados, dos quais
68.735 frequentavam habitualmente as aulas, portanto com chances de serem pesquisados. No dia da coleta dos dados, 63.411 estavam presentes, representando perdas de $7,7 \%$ nesta fase. Desses últimos, 501 estudantes não quiseram responder ao questionário da pesquisa e 1.937 não informaram o sexo e foram excluídos das tabulações. Assim, a amostra da PeNSE totalizou 60.973 estudantes de 1.453 escolas públicas e privadas nas 27 capitais, totalizando taxa de resposta de $88,7 \%$. A maioria tinha de 13 a 15 anos (89\%).

Para o presente estudo, foram selecionados apenas os estudantes que responderam "sim" ou "não" à questão sobre dor de dente, sendo excluídos os 5.988 que responderam "Não sei” ou que não responderam esta questão. Dessa forma, dos 60.973 estudantes que responderam ao questionário da PeNSE, um total de 54.985 foi incluído na presente investigação. A idade variou de 11 a 19 anos ou mais e 53,6\% eram do sexo feminino.

A prevalência de dor de dente foi de $17,8 \%$ (IC95\%: 17,5-18,1) para a amostra como um todo. Os valores mais elevados foram encontrados em capitais situadas nas regiões Norte e Centrooeste, com exceção de uma da Região Sul. As prevalências variaram de 13,7\% em Vitória a 22,6\% em Boa Vista (Figura 2).

As frequências das categorias de variáveis demográficas, condições socioeconômicas e de comportamentos associados à saúde geral e bucal estão descritas nas Tabelas 1 e 2. A Tabela 1 mostra que a prevalência de dor dentária foi significativamente maior em escolares do sexo feminino (19,7\%), nas faixas etárias de 14 a 16 anos (17,9\%) e maior que 17 anos (22,9\%), e nos adolescentes que se classificaram como pardos 


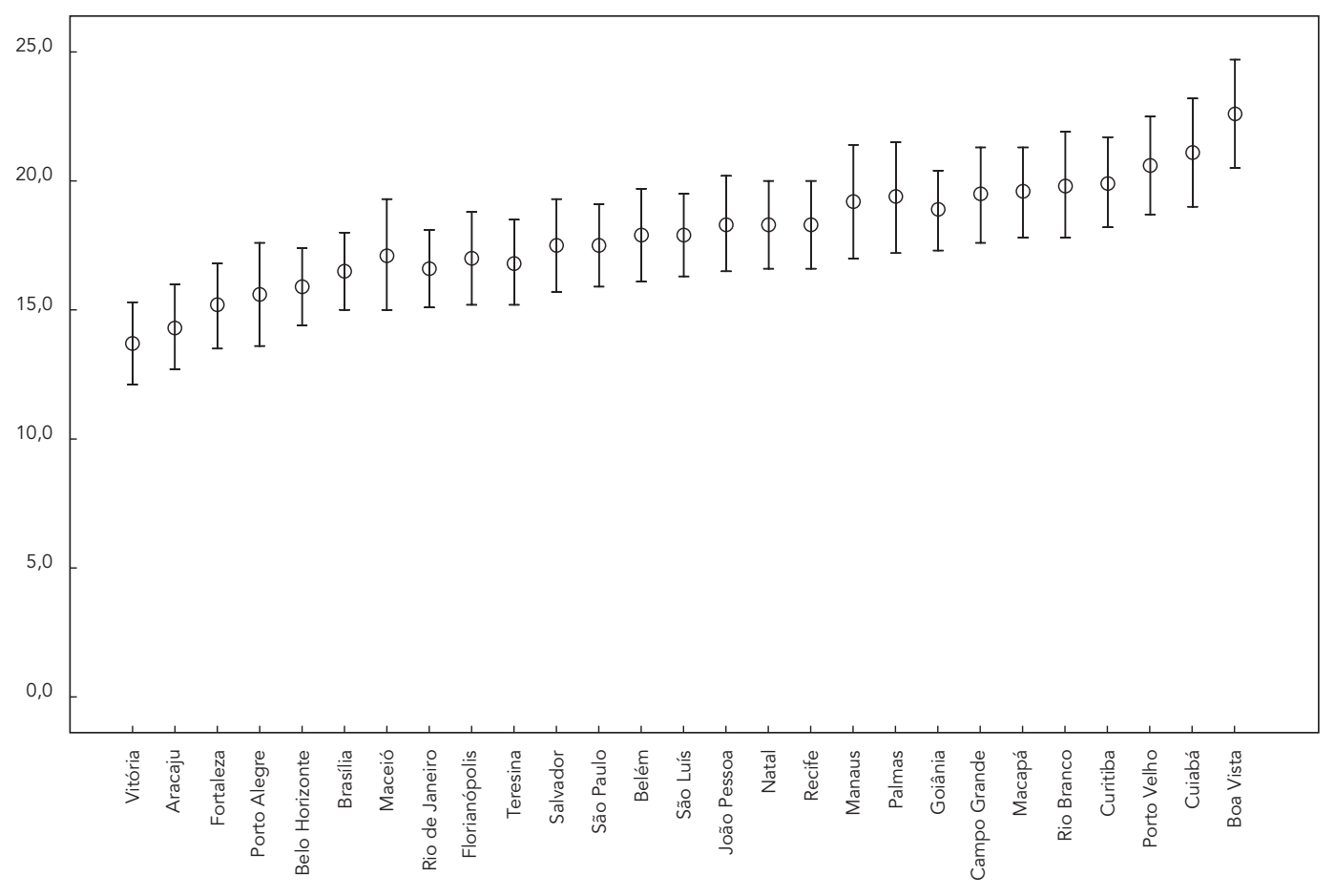

(18,3\%), pretos (20\%) e indígenas $(20,6 \%)$. A escolaridade da mãe foi inversamente associada à prevalência de dor dentária, e a maior prevalência foi encontrada em estudantes de escola pública $(18,7 \%)$ e que não moram com a mãe $(20,3 \%)$. Todos os comportamentos desfavoráveis em relação à saúde geral e bucal foram associados à maior prevalência de dor dentária.

As medidas da razão de prevalência não ajustadas (Tabelas 1 e 2) mostram as variáveis relacionadas com a dor de dente. A análise de regressão múltipla de Poisson (Tabela 3) mostrou que a ocorrência de dor dentária foi associada ao sexo feminino, idades acima de 14 anos, raças preta, parda e indígena, e condições socioeconômicas desfavoráveis como a baixa escolaridade materna, não morar com a mãe e estudantes de escolas públicas. Hábitos e comportamentos negativos em relação à saúde como o tabagismo, experiência de consumo de álcool, baixos níveis de atividade física, baixa frequência de escovação diária e consumo frequente de guloseimas e refrigerantes foram associados à ocorrência de dor de dente.
Após ajuste das variáveis do bloco 1, todas as variáveis analisadas apresentaram associação com a dor de dente. Da mesma forma, todas as variáveis permaneceram associadas no bloco 2 e a variável tempo de atividade física acumulada foi removida no bloco 3. Dentre todas as variáveis incluídas no modelo final, a maior associação com a ocorrência de dor de dente foi observada em escolares com baixa frequência de escovação diária (RP = 1,82; IC95\%: 1,60-2,06), sendo a prevalência $80 \%$ maior em relação àqueles que relataram frequência de escovação de 3 vezes ou mais ao dia. A prevalência de dor de dente foi $34 \%$ maior no sexo feminino, 33\% maior em estudantes de escolas públicas e $29 \%$ maior entre aqueles que relataram ter experimentado cigarro alguma vez na vida. Em relação aos grupos raciais, pretos e indígenas apresentaram prevalência de dor de dente $29 \%$ e $32 \%$ maior, respectivamente, em relação aos brancos. 
Tabela 1

Distribuição de frequências e prevalência de dor de dente segundo variáveis demográficas e socioeconômicas, em adolescentes das capitais brasileiras Pesquisa Nacional de Saúde do Escolar (PeNSE), Brasil, 2009.

\begin{tabular}{|c|c|c|c|c|}
\hline Variáveis & $\begin{array}{l}\text { Frequência } \\
\text { (\% válidos) }\end{array}$ & $\begin{array}{l}\text { Prevalência } \\
\%(I C 95 \%) *\end{array}$ & $\begin{array}{l}\text { RP não ajustada } \\
\text { (IC95\%) * }\end{array}$ & Valor de $p$ ** \\
\hline \multicolumn{5}{|l|}{ Demográficas } \\
\hline \multicolumn{5}{|c|}{ Sexo $[n=54.985]$} \\
\hline Masculino & $25.512(46,4)$ & $14,9(14,1-15,7)$ & Referência & $<0,001$ \\
\hline Feminino & $29.473(53,6)$ & $19,7(18,9-20,5)$ & $1,32(1,24-1,41)$ & \\
\hline \multicolumn{5}{|c|}{ Idade (anos) $[\mathrm{n}=54.871]$} \\
\hline $11-13$ & $14.164(25,8)$ & $15,5(14,4-16,6)$ & Referência & $<0,001$ \\
\hline $14-16$ & $38.666(70,5)$ & $17,9(17,2-18,5)$ & $1,15(1,06-1,25)$ & \\
\hline$>17$ & $2.040(3,7)$ & $22,9(20,3-25,6)$ & $1,48(1,29-1,69)$ & \\
\hline \multicolumn{5}{|c|}{ Raça [n = 53.994] } \\
\hline Branca & $19.923(36,9)$ & $15,4(14,5-16,3)$ & Referência & $<0,001$ \\
\hline Parda & $22.738(42,1)$ & $18,3(17,4-19,2)$ & $1,19(1,10-1,28)$ & \\
\hline Preta & $6.749(12,5)$ & $20,0(18,4-21,7)$ & $1,30(1,18-1,44)$ & \\
\hline Amarela & $2.210(4,1)$ & $18,0(15,5-20,6)$ & $1,17(1,00-1,36)$ & \\
\hline Indígena & $2.374(4,4)$ & $20,6(17,8-23,3)$ & $1,33(1,15-1,54)$ & \\
\hline \multicolumn{5}{|c|}{ Socioeconômicas } \\
\hline \multicolumn{5}{|c|}{ Escolaridade da mãe (anos) [n = 45.239] } \\
\hline$>11$ & $12.118(26,8)$ & $13,8(12,7-14,9)$ & Referência & $<0,001$ \\
\hline $8-11$ & $19.146(42,3)$ & $17,5(16,5-18,4)$ & $1,27(1,15-1,40)$ & \\
\hline$<8$ & $13.975(30,9)$ & $20,6(19,5-21,8)$ & $1,50(1,36-1,65)$ & \\
\hline \multicolumn{5}{|c|}{ Mora com a mãe [n = 54.298] } \\
\hline Sim & $48.467(89,3)$ & $17,2(16,6-17,7)$ & Referência & $<0,001$ \\
\hline Não & $5.831(10,7)$ & $20,3(18,5-22,1)$ & $1,18(1,08-1,30)$ & \\
\hline \multicolumn{5}{|c|}{ Tipo de escola $[n=54.985]$} \\
\hline Privada & $13.162(23,9)$ & $12,8(11,8-13,7)$ & Referência & $<0,001$ \\
\hline Pública & $41.823(76,1)$ & $18,7(18,0-19,3)$ & $1,47(1,35-1,59)$ & \\
\hline
\end{tabular}

IC95\%: intervalo de 95\% de confiança; RP: razão de prevalência.

* Corrigida para o desenho amostral;

** Teste de Wald.

\section{Discussão}

O presente trabalho é o primeiro sobre dor de dente em adolescentes brasileiros utilizando dados de um inquérito nacional realizado em amostra representativa de escolares no conjunto das capitais brasileiras. Um dos pontos relevantes desta abordagem é o tamanho da amostra e a qualidade do instrumento, contendo questões validadas sobre fatores de risco e proteção para a saúde, as quais não têm sido incluídas nos levantamentos nacionais de saúde bucal.

O fato de constituir um estudo de base escolar tem implicações que devem ser discutidas na presente pesquisa. A população escolar exclui os jovens que estão fora da escola. Contudo, no Brasil, a cobertura do sistema de ensino tem sido alta e a grande maioria dos adolescentes entre 10 e 14 anos (97\%) encontrava-se matriculada em escolas em 2007 24, ano que serviu de base para o cálculo amostral da PeNSE. Outra vantagem de realizar a pesquisa no ambiente escolar consiste na possibilidade dos resultados serem utilizados na indução de políticas públicas de promoção à saúde e prevenção para o público específico.

A prevalência de dor de dente nos últimos seis meses $(17,8 \%)$ foi menor do que a verificada em adolescentes brasileiros de 15 a 19 anos em 2003 (35,7\%) 16,17 e em escolares dos municípios de São Paulo (25,6\% aos 12 e 15 anos) 7 e do Recife (33,6\% aos 14 e 15 anos) 19 , nos quais foi utilizado o mesmo tempo recordatório. Da mesma forma, quando analisados separadamente, os porcentuais de relato de dor dessas duas capitais 
Distribuição de frequências e prevalência de dor de dente segundo hábitos e presença de fatores associados à cárie dentária, em adolescentes das capitais brasileiras. Pesquisa Nacional de Saúde do Escolar (PeNSE), Brasil, 2009.

\begin{tabular}{|c|c|c|c|c|}
\hline Variável & $\begin{array}{l}\text { Frequência } \\
\text { (\% válidos) }\end{array}$ & $\begin{array}{l}\text { Prevalência } \\
\%(I C 95 \%) *\end{array}$ & $\begin{array}{l}\text { RP não ajustada } \\
\text { (IC95\%) * }\end{array}$ & Valor de $p$ ** \\
\hline \multicolumn{5}{|l|}{ Comportamentos associados à saúde } \\
\hline \multicolumn{5}{|l|}{ Fumou alguma vez [n = 54.220] } \\
\hline Não & $41.660(76,8)$ & $15,7(15,1-16,3)$ & Referência & $<0,001$ \\
\hline $\operatorname{Sim}$ & $12.560(23,2)$ & $23,2(21,9-24,5)$ & $1,48(1,38-1,58)$ & \\
\hline \multicolumn{5}{|l|}{ Consumo de álcool [n = 54.252] } \\
\hline Não & $16.705(30,8)$ & $13,9(13,0-14,8)$ & Referência & $<0,001$ \\
\hline Sim & $37.547(69,2)$ & $18,9(18,2-19,6)$ & $1,36(1,26-1,46)$ & \\
\hline \multicolumn{5}{|l|}{$\begin{array}{l}\text { Tempo de atividade física acumulada nos últimos } \\
\text { sete dias [ } n=54.985]\end{array}$} \\
\hline Ativo & $23.393(42,5)$ & $16,1(15,3-16,9)$ & Referência & $<0,001$ \\
\hline Insuficientemente ativo (150-299 minutos) & $14.699(26,8)$ & $17,5(16,5-18,6)$ & $1,09(1,01-1,18)$ & \\
\hline Insuficientemente ativo ( $\leq 149$ minutos) & $14.172(25,8)$ & $19,2(18,0-20,3)$ & $1,19(1,10-1,29)$ & \\
\hline Inativo & $2.721(4,9)$ & $20,4(17,7-23,0)$ & $1,27(1,10-1,45)$ & \\
\hline \multicolumn{5}{|l|}{ Comportamentos associados à saúde bucal } \\
\hline \multicolumn{5}{|l|}{ Frequência diária de escovação [n = 54.197] } \\
\hline 3 ou mais vezes & $40.552(74,8)$ & $16,5(15,9-17,2)$ & Referência & $<0,001$ \\
\hline 2 vezes & $11.197(20,7)$ & $18,0(16,7-19,2)$ & $1,09(1,00-1,17)$ & \\
\hline $0-1$ vez & $2.448(4,5)$ & $29,5(26,3-32,7)$ & $1,78(1,59-2,00)$ & \\
\hline \multicolumn{5}{|l|}{ Consumo semanal de guloseimas $[n=54.233]$} \\
\hline Baixo & $27.691(51,1)$ & $15,8(15,1-16,6)$ & Referência & $<0,001$ \\
\hline Alto & $26.542(48,9)$ & $19,0(18,2-19,8)$ & $1,20(1,13-1,28)$ & \\
\hline \multicolumn{5}{|l|}{ Consumo semanal de refrigerantes $[n=54.267]$} \\
\hline Baixo & $35.054(64,6)$ & $16,4(15,8-17,1)$ & Referência & $<0,001$ \\
\hline Alto & $19.213(35,4)$ & $19,1(18,2-20,1)$ & $1,16(1,09-1,24)$ & \\
\hline
\end{tabular}

IC95\%: intervalo de 95\% de confiança; RP: razão de prevalência.

* Corrigida para o desenho amostral;

** Teste de Wald.

foram mais baixos no presente estudo, estando abaixo de 20\%. Essas diferenças eram esperadas, considerando-se que na amostra nacional foram também incluídos escolares mais jovens e mais velhos, e ao fato de que as capitais não são homogêneas em relação ao nível de desenvolvimento econômico e à condição de saúde bucal, o que influencia a média nacional.

Comparações com os resultados dos demais estudos realizados no Brasil também não permitem conclusões precisas, pois diferentes idades têm sido incluídas nas amostras e o relato da dor tem sido obtido em diferentes períodos de tempo. Variações nas metodologias utilizadas podem ser observadas também nos trabalhos internacionais. Em Kampala, Uganda, 47,6\% dos escolares de 10 a 14 anos relataram dor de dente nos últimos 12 meses 32 . No Paquistão, 30,4\% dos estudantes de 11 a 14 anos 33, e em Toronto, Cana- dá, 18\% dos adolescentes de 14 a 20 anos tiveram dor no último mês 34 . Na Tanzânia, a prevalência nos últimos três meses foi de $36,4 \%$ entre alunos de 10 a 19 anos de escolas públicas 9 .

A associação verificada entre dor de dente e todos os fatores analisados no presente estudo, e a consistência da maioria dos achados com a evidência científica disponível chamam a atenção. Após a regressão múltipla, somente a variável atividade física não permaneceu estatisticamente significante. Maior prevalência de relato de dor de dente foi associada às condições adversas de vida e à maior exposição aos comportamentais de risco à saúde, corroborando os resultados de estudos anteriores 10 .

A relação encontrada entre maior prevalência de dor de dente e condições socioeconômicas desfavoráveis, assim como os percentuais mais elevados nas capitais das regiões menos favore- 
Tabela 3

Resultado da análise de regressão de Poisson da associação entre dor de dente e variáveis independentes, em adolescentes das capitais brasileiras. Pesquisa Nacional de Saúde do Escolar (PeNSE), Brasil, 2009.

\begin{tabular}{|c|c|c|c|c|}
\hline Blocos & Variáveis & $\begin{array}{c}\text { Modelo I } \\
\text { [RP (IC95\%)] }\end{array}$ & $\begin{array}{c}\text { Modelo II } \\
\text { [RP (IC95\%)] }\end{array}$ & $\begin{array}{c}\text { Modelo III } \\
\text { [RP (IC95\%)] }\end{array}$ \\
\hline \multirow[t]{13}{*}{1} & Sexo $[p<0,001]$ & & & \\
\hline & Masculino & 1,00 & & \\
\hline & Feminino & $1,34(1,25-1,43)$ & & \\
\hline & Idade (anos) $[p<0,001]$ & & & \\
\hline & $11-13$ & 1,00 & & \\
\hline & $14-16$ & $1,16(1,07-1,26)$ & & \\
\hline & $>17$ & $1,46(1,27-1,68)$ & & \\
\hline & Raça $[p<0,001]$ & & & \\
\hline & Branca & 1,00 & & \\
\hline & Parda & $1,16(1,07-1,25)$ & & \\
\hline & Preta & $1,29(1,17-1,43)$ & & \\
\hline & Amarela & $1,14(0,98-1,32)$ & & \\
\hline & Indígena & $1,32(1,14-1,53)$ & & \\
\hline \multirow[t]{10}{*}{2} & Escolaridade da mãe (anos) $[p=0,001]$ & & & \\
\hline & $>11$ & & 1,00 & \\
\hline & $8-11$ & & $1,10(0,98-1,22)$ & \\
\hline & $<8$ & & $1,20(1,07-1,35)$ & \\
\hline & Mora com a mãe $[p=0,003]$ & & & \\
\hline & $\operatorname{Sim}$ & & 1,00 & \\
\hline & Não & & $1,18(1,06-1,32)$ & \\
\hline & Tipo de escola $[p<0,001]$ & & & \\
\hline & Privada & & 1,00 & \\
\hline & Pública & & $1,33(1,19-1,47)$ & \\
\hline \multirow[t]{16}{*}{3} & Fumou alguma vez $[p<0,001]$ & & & \\
\hline & Não & & & 1,00 \\
\hline & Sim & & & $1,29(1,15-1,38)$ \\
\hline & Consumo de álcool [p < 0,001] & & & \\
\hline & Não & & & 1,00 \\
\hline & $\operatorname{Sim}$ & & & $1,26(1,15-1,38)$ \\
\hline & Frequência diária de escovação [p $<0,001]$ & & & \\
\hline & 3 ou mais vezes & & & 1,00 \\
\hline & 2 vezes & & & $1,14(1,04-1,25)$ \\
\hline & $0-1$ vez & & & $1,82(1,60-2,06)$ \\
\hline & Consumo de guloseimas $[p=0,003]$ & & & \\
\hline & Baixo & & & 1,00 \\
\hline & Alto & & & $1,12(1,04-1,21)$ \\
\hline & Consumo de refrigerantes $[p=0,001]$ & & & \\
\hline & Baixo & & & 1,00 \\
\hline & Alto & & & $1,13(1,05-1,22)$ \\
\hline
\end{tabular}

IC95\%: intervalo de 95\% de confiança; RP: razão de prevalência.

Nota 1: Modelos I a III: razão de prevalência ajustada pelas variáveis do mesmo bloco e acima.

Nota 2: razão de prevalência corrigida para o desenho amostral.

cidas economicamente, confirmam a influência da desigualdade social na saúde bucal da população brasileira. A grande maioria dos estudos publicados sobre a dor de dente na infância e na adolescência no Brasil mostra o efeito de um ou mais fatores socioeconômicos, geralmente utili- 
zando como indicadores a classificação socioeconômica das famílias, a renda per capita, a escolaridade dos pais ou da mãe, e o tipo de escola (pública e privada) 7,13,17,18,19,20,35. Essa influência ocorre também em relação a variáveis contextuais. Utilizando análise multinível, Peres et al. 7 verificaram ainda que escolares de São Paulo que viviam em áreas com Índice de Desenvolvimento Humano (IDH) mais baixo apresentavam maior prevalência de dor de dente do que aqueles de áreas mais desenvolvidas, independentemente das características individuais. A relação entre privação econômica e dor de dente pode ser resultado da maior exposição dos menos privilegiados aos fatores de risco para os problemas bucais, tais como a dieta cariogênica, as deficiências no estado imunitário e nutricional, e o baixo acesso aos fluoretos, recursos de higiene bucal e serviços de saúde 11. Outra hipótese é que os indivíduos menos favorecidos economicamente estariam mais expostos a eventos estressantes e poderiam apresentar menor limiar para dor 11.

Não há consenso na literatura sobre a associação entre gênero e dor de dente. Maior prevalência entre indivíduos do sexo feminino foi verificada neste e em outros estudos, tanto em adolescentes 7,17,35 como em adultos 6,31. Outros pesquisadores, entretanto, não encontraram essa associação 13,18,19,32,36. Em um grupo de adultos, ser do sexo masculino aumentou a chance de ter dor de dente que impediu a realização de tarefas habituais 37 . Estudos experimentais mostram que as mulheres são mais sensíveis à dor provocada, contudo a magnitude dos achados tem sido criticada por serem baseados em tamanhos reduzidos de amostra 38. Em qualquer análise deve-se considerar que a dor é um fenômeno complexo, que pode ser influenciado por fatores biológicos, culturais e psicológicos, pelas expectativas em relação aos papéis sociais, por fatores situacionais e pela história passada do indivíduo 38,39,40.

A presença do pai biológico na família tem sido pouco investigada. Em Pelotas (Rio Grande do Sul), foi encontrada associação entre dor de dente em crianças aos 6 e aos 12 anos e a presença do pai ao nascimento ${ }^{35}$. Na Grécia, os resultados foram similares na idade de 12 anos 41 . Os mecanismos pelos quais essa influência ocorre não estão esclarecidos, mas podem estar relacionados à maior privação econômica das famílias constituídas por um único pai ou mãe biológico. Contudo, quando controlados por condições socioeconômicas essa associação se manteve. Fatores relacionados aos aspectos psicossociais desse tipo de estrutura familiar também devem ser considerados, pois podem influenciar no cuidado com a saúde.
A variável raça ou cor da pele tem sido incluída em poucos estudos sobre dor de dente, cujos resultados são conflitantes em adolescentes 7,17 e em adultos 6,31,37. No presente trabalho, adolescentes das raças preta, parda e indígena, que geralmente constituem grupos de condição socioeconômica inferior, apresentaram mais relatos de dor do que aqueles da raça branca. A relação entre raça preta e dor de dente pode ser em decorrência da maior prevalência de problemas de saúde bucal neste grupo 42 .

Existe evidência de que a dor de dente é mais frequente em indivíduos com experiência de cárie mais elevada 7,13,17,18,19,21,32,35,36. Não obstante, na Finlândia houve aumento do relato de odontalgia entre adolescentes apesar do declínio de cárie nos anos 1990, sugerindo deficiências no sistema de saúde daquele país 43 . Para o presente estudo, cujo instrumento não foi planejado especificamente para pesquisas sobre saúde bucal, não se encontravam disponíveis variáveis clínicas sobre a condição de saúde bucal.

A inclusão das variáveis higiene bucal e consumo de alimentos que contêm açúcar teve como finalidade verificar se estes hábitos relacionados à condição de saúde bucal, em especial à cárie dentária, apresentam associação com o relato de dor. Ambas foram significantes mesmo após o ajuste pelas demais variáveis. Quanto à frequência de escovação, o resultado deste estudo diverge dos anteriores em crianças e adolescentes 18,35. Contudo, está de acordo com os resultados encontrados em adolescentes finlandeses, em que a frequência de escovação foi o fator mais fortemente associado com experiência de odontalgia 43.

Somente um estudo sobre dor de dente investigou a dieta dos indivíduos, e os resultados mostraram que não há associação significante entre dor e a presença de açúcar no café da manhã, no almoço e no jantar, em escolares de 12 e 13 anos 18 . Porém, a variável utilizada refere-se apenas às principais refeições e sabe-se que a cárie está associada ao consumo frequente de açúcar, que inclui os momentos entre as refeições ${ }^{44}$. A alta frequência de consumo semanal de alimentos e bebidas que contêm açúcares na população adolescente, revelada pela PeNSE, é preocupante, pois constitui um importante fator de risco para as doenças crônicas não transmissíveis, além de ter um papel fundamental na etiologia da cárie dentária 23,44.

Este é o primeiro estudo no Brasil a investigar o uso de cigarro e de álcool em relação à dor de dente em adolescentes. Na literatura há evidências de associação entre essas variáveis na população adulta no Brasil 6,31 e na América do Norte 45,46. As prováveis explicações para o maior risco 
de dor entre fumantes são as doenças periodontais, a redução do fluxo salivar e a cárie, decorrentes do uso de tabaco 6,31,46. A coexistência de diversos fatores de risco entre adolescentes, como uso de álcool, tabaco, drogas, dentre outros, tem sido descrita em outros estudos de associação da PeNSE e também da literatura, o que poderia explicar a concomitância do uso do álcool, tabaco e da dor encontrada neste estudo 47,48 .

O uso mais frequente de álcool entre os adolescentes com maior relato de dor de dente pode ser interpretado como um recurso para lidar com este sintoma, buscando reduzir o estresse causado pela dor. Essa questão tem sido objeto de pesquisas realizadas com adultos e idosos nos Estados Unidos 49,50,51. O consumo de álcool está associado também à depressão e ao uso de medicamentos com ou sem prescrição entre adultos com dor de origem dental ou facial 3,51 .

A atividade física foi incluída na análise como indicador de impacto no desempenho das atividades dos adolescentes, baseando-se na hipótese de que a dor de dente causaria redução neste tipo de atividade. Contudo, a associação verificada entre as duas variáveis foi influenciada pelas demais e não se manteve significativa no modelo final. Não foram encontrados na literatura outros estudos sobre essa questão.

Os resultados aqui apresentados devem ser interpretados levando-se em consideração o tipo de delineamento do trabalho, pois estudos transversais não permitem conclusões sobre a relação causa-efeito. Outra limitação é o fato da amostra ser composta por adolescentes somente das capitais brasileiras. Municípios do interior do país, assim como aqueles de menor porte populacional incluídos no levantamento nacional de saúde bucal de 2003, apresentaram maior experiência de cárie ${ }^{52}$, menor cobertura de água fluoretada e, considerando a relação desta com a dor de dente, pode ser que apresentem também maiores prevalências em comparação com as capitais. A dor de dente constitui um importante indicador subjetivo de saúde bucal, e o seu monitoramento em relação à tendência da cárie nos levantamentos nacionais e locais deve ser parte da estratégia de vigilância em saúde bucal no país.

Conclui-se que a prevalência de dor de dente na população estudada foi considerável e associada a aspectos demográficos, socioeconômicos e de comportamentos relacionados à saúde geral e bucal. Isso significa que estratégias voltadas para a melhoria das condições de vida e a promoção de comportamentos saudáveis têm o potencial de reduzir a prevalência dos problemas bucais que produzem dor de dente e impacto na qualidade de vida dos pesquisados. Como o estudo foi realizado em escolas, medidas nestas instituições buscando reduzir o sofrimento causado pelos sintomas e seu provável impacto no rendimento escolar devem ser consideradas, especialmente nas escolas públicas, onde a prevalência da dor de dente foi mais elevada. Nesse sentido, os dados do presente estudo podem contribuir para embasar as ações como as do Programa de Saúde na Escola 53.

Além disso, dentro da abordagem dos fatores de risco comuns, os principais problemas de saúde bucal estão associados aos mesmos fatores de risco para as doenças crônicas, tais como a obesidade, o diabetes, as doenças cardiovasculares e o câncer ${ }^{54}$. Assim, os dados deste trabalho justificam ainda intervenções focadas na prevenção e redução concomitante dos principais fatores comportamentais de risco de doenças não transmissíveis na população em geral. 


\section{Resumo}

O objetivo deste estudo foi estimar a prevalência da dor de dente em adolescentes brasileiros e analisar fatores sociodemográficos e comportamentais associados, utilizando os dados da Pesquisa Nacional de Saúde do Escolar (PeNSE) de 2009. A pesquisa foi realizada pelo Instituto Brasileiro de Geografia e Estatística e pelo Ministério da Saúde em escolares com idades entre 11 e 17 anos ou mais, das 27 capitais brasileiras, por meio de questionário autoaplicável. Utilizou-se a análise de regressão de Poisson, segundo um modelo hierárquico de determinação. A prevalência de dor na amostra ( $n=54.985)$ nos últimos seis meses foi de 17,8\% (IC95\%: 17,5-18,1). Prevalências mais elevadas foram encontradas em mulheres, naqueles com 14 anos ou mais, das raças preta, parda e indígena, de escolas públicas, cujas mães tinham baixa escolaridade, que não moravam com a mãe, que haviam experimentado cigarro $e$ álcool alguma vez na vida, que relataram menor frequência de escovação e maior consumo de guloseimas e refrigerantes. A prevalência de dor foi considerável e associada a aspectos sociodemográficos e de comportamentos relacionados à saúde.

Odontalgia; Fatores de Risco; Fatores Socioeconômicos; Saúde Bucal

\section{Colaboradores}

M. C. M. Freire foi responsável pelo planejamento do estudo, colaborou na elaboração da análise dos dados e na redação do artigo, e aprovou a versão final. C. R. Leles colaborou no planejamento do estudo, realizou a análise e interpretação dos dados, colaborou na redação do artigo, e aprovou a versão final. L. M. V. Sardinha, M. Paludetto Junior e D. C. Malta colaboraram na redação do artigo e aprovaram a versão final. M. A. Peres participou do planejamento do estudo, colaborou na elaboração da análise dos dados e sua interpretação, realizou a revisão crítica do artigo e aprovou a versão final.

\section{Agradecimentos}

Ao Ministério da Saúde.

\section{Referências}

1. Goes PSA, Kosminsky M, Siqueira JTT, Ribeiro MFP Dor orofacial. In: Antunes JLP, Peres MA, organizadores. Epidemiologia da saúde bucal. Rio de Janeiro: Editora Guanabara Koogan; 2006. p. 102-14.

2. Locker D, Grushka M. The impact of dental and facial pain. J Dent Res 1987; 66:1414-7.

3. Silva RA, Marques FD, Goes PSA. Fatores associados à automedicação em dor de dente: análise a partir dos profissionais dos estabelecimentos farmacêuticos da Cidade do Recife, PE. Ciênc Saúde Coletiva 2008; 13 Suppl:697-701.
4. Luzzi L, Jones K, Spencer AJ, Roberts-Thomson KF. Association of urgent dental care with subjective oral health indicators and psychosocial impact. Community Dent Health 2009; 26:77-83.

5. Hobdell M, Petersen PE, Clarkson J, Johnson N. Global goals for oral health 2020. Int Dental J 2003; 53:285-8.

6. Bastos JL, Gigante DP, Peres KG. Toothache prevalence and associated factors: a population based study in southern Brazil. Oral Dis 2008; 14:320-6. 
7. Peres MA, Peres KG, Frias AC, Antunes JLF. Contextual and individual assessment of dental pain period prevalence in adolescents: a multilevel approach. BMC Oral Health 2010; 10:20.

8. Goes PSA, Watt RG, Hardy R, Sheiham A. Impacts of dental pain on daily activities of adolescents aged 14-15 years and their families. Acta Odontol Scand 2008; 66:7-12.

9. Mashoto KO, Åstrøm NA, David J, Masalu JR. Dental pain, oral impacts and perceived need for dental treatment in Tanzanian school students: a cross-sectional study. Health Qual Life Outcomes 2009; 7:73.

10. Pau AK, Croucher R, Marcenes W. Prevalence estimates and associated factors for dental pain: a review. Oral Health Prev Dent 2003; 1:209-20.

11. Bastos JLD, Gigante DP, Peres KG, Nedel FB. Determinação social da odontalgia em estudos epidemiológicos: revisão teórica e proposta de um modelo conceitual. Ciênc Saúde Coletiva 2007; 12:1611-21.

12. Shepherd MA, Nadanovsky P, Sheiham A. The prevalence and impact of dental pain in 8-yearold school children in Harrow, England. Br Dent J 1999; 187:38-41.

13. Barrêtto EPR, Ferreira EF, Pordeus IA. Determinant factors of toothache in 8- and 9-year-old schoolchildren, Belo Horizonte, MG, Brazil. Braz Oral Res 2009; 23:124-30.

14. Nuttall NM, Steele JG, Evans D, Chadwick B, Morris AJ, Hill K. The reported impact of oral condition on children in the United Kingdom, 2003. Br Dent J 2006; 200:551-6.

15. Lewis C, Stout J. Toothache in US children. Arch Pediatr Adolesc Med 2010; 164:1059-63.

16. Coordenação Nacional de Saúde Bucal, Departamento de Atenção Básica, Secretaria de Atenção à Saúde, Ministério da Saúde. Projeto SB Brasil 2003. Condições de saúde bucal da população brasileira 2002-2003: resultados principais. Brasília: Ministério da Saúde; 2004. (Série C. Projetos, Programas e Relatórios)

17. Borges CM, Cascaes AM, Fischer TK, Boing AF, Peres MA, Peres KG. Dor nos dentes e gengivas e fatores associados em adolescentes brasileiros: análise do inquérito nacional de saúde bucal SB-Brasil 2002-2003. Cad Saúde Pública 2008; 24:1825-34.

18. Nomura LH, Bastos JLD, Peres MA. Dental pain prevalence and association with dental caries and socioeconomic status in schoolchildren, Southern Brazil, 2002. Braz Oral Res 2004; 18:134-40.

19. Goes PS, Watt R, Hardy RG, Sheiham A. The prevalence and severity of dental pain in 14-15 year old Brazilian schoolchildren. Community Dent Health 2007; 24:217-24.

20. Hack-Comunello SM, Michel-Crosato E, Biazevic MGH, Crosato E. Dor dental e condição sócio-econômica: um estudo censitário em escolares. Pesqui Bras Odontopediatria Clín Integr 2008; 8:63-7.

21. Rihs LB, Cypriano S, Sousa MLR, Silva RC, Gomes PR. Dor de dente e sua relação com a experiência de cárie em adolescentes. RGO (Porto Alegre) 2008; 56:361-5.
22. Instituto Brasileiro de Geografia e Estatística. Pesquisa Nacional de Saúde do Escolar (PeNSE) 2009. http://www.ibge.gov.br/home/estatistica/ populacao/pense/default.shtm (acessado em 10/ Jan/2010).

23. Malta DC, Sardinha LMV, Mendes I, Barreto SM, Giatti L, Castro IRR, et al. Prevalência de fatores de risco e proteção de doenças crônicas não transmissíveis em adolescentes: resultados da Pesquisa Nacional de Saúde do Escolar (PeNSE), Brasil, 2009. Ciênc Saúde Coletiva 2010; 15:3009-19.

24. Instituto Brasileiro de Geografia e Estatística. Pesquisa Nacional por Amostra de Domicílios 2008. v. 29. http://www.ibge.gov.br/home/estatistica/po pulacao/trabalhoerendimento/pnad2008/brasil pnad2008.pdf (acessado em 20/Jul/2010).

25. Instituto Nacional de Estudos e Pesquisas Educacionais Anísio Teixeira. Censo Escolar 2007. http:// www.inep.gov.br/basica/saeb/matrizes.htm (acessado em 25/Mar/2010).

26. Victora CG, Barros FC, Martines JC, Béria JU, Vaughan JP. Do mothers remember their children's birth weights? Rev Saúde Pública 1985; 19:58-68.

27. Instituto Nacional de Câncer. Vigescola: vigilância de tabagismo em escolares - dados e fatos de 12 capitais brasileiras 2004. http://www.inca.gov.br/ vigescola/ (acessado em 13/Ago/2010).

28. Castro IRR, Cardoso LO, Engstrom EM, Levy RB, Monteiro CA. Vigilância de fatores de risco para doenças não transmissíveis entre adolescentes: a experiência da Cidade do Rio de Janeiro, Brasil. Cad Saúde Pública 2008; 24:2279-88.

29. Brener ND, Kann L, Kinchen SA, Grunbaum AJ, Whalen L, Eaton D, et al. Methodology of the youth risk behavior surveillance system. MMWR Recomm Rep 2004; 53:1-13.

30. World Health Organization. Inequalities young people's health: key findings from the Health Behaviour in School-aged Children (HBSC) 2005/2006 survey fact sheet. Copenhagen: World Health Organization; 2008.

31. Kuhnen M, Peres MA, Masiero AV, Peres KG. Toothache and associated factors in Brazilian adults: a cross-sectional population-based study. BMC Oral Health 2009; 9:7.

32. Kiwanuka SN, Åstrøm AN. Self-reported dental pain and associated factors in Ugandan schoolchildren. Norsk Epidemiologi 2005; 15:175-82.

33. Pau A, Khan SS, Babar MG, Croucher R. Dental pain and care-seeking in 11-14-yr-old adolescents in a low-income country. Eur J Oral Sci 2008; 116:451-7.

34. Clarke M, Locker D, Murray H, Payne B. The oral health of disadvantaged adolescents in North York, Ontario. Can J Public Health 1996; 4:261-3.

35. Bastos JL, Peres MA, Peres KG, Araujo CLP, Menezes AMB. Toothache prevalence and associated factors: a life course study from birth to age $12 \mathrm{yr}$. Eur J Oral Sci 2008; 116:458-66.

36. Lacerda JT, Simionato EM, Peres KG, Peres MA, Traebert J, Marcenes W. Dor de origem dental como motivo de consulta odontológica em uma população adulta. Rev Saúde Pública 2004; 38:453-8. 
37. Alexandre GC, Nadanovsky P, Lopes CS, Faerstein E. Prevalência e fatores associados à ocorrência da dor de dente que impediu a realização de tarefas habituais em uma população de funcionários públicos no Rio de Janeiro, Brasil. Cad Saúde Pública 2006; 22:1073-8.

38. Riley JL, Robinson ME, Wise EA, Myers CD, Fillingim RB. Sex differences in the perception of noxious experimental stimuli: a meta-analysis. Pain 1998; 74:181-7.

39. Liddell A, Locker D. Gender and age differences in attitudes to dental pain and dental control. Community Dent Oral Epidemiol 1997; 25:314-8.

40. Dao TT, LeResche L. Gender differences in pain. J Orofac Pain 2000; 14:169-84.

41. Pau A, Baxevanos KG, Croucher R. Family structure is associated with oral pain in 12-year-old Greek school children. Int J Paediatr Dent 2007; 17:345-51.

42. Bastos JL, Antunes JLF, Frias AC, Souza MLR, Peres KG, Peres MA. Color/race inequalities in oral health among Brazilian adolescents. Rev Bras Epidemiol 2009; 12:313-24.

43. Honkala E, Honkala S, Rimpelä A, Rimpelä M. The trend and risk factors of perceived toothache among Finnish adolescents from 1977 to 1997. J Dent Res 2001; 80:1823-7.

44. Freire MCM. Políticas públicas de alimentação saudável. In: Bonecker M, Sheiham A, organizadores. Promovendo saúde bucal na infância e adolescência: conhecimentos e práticas. São Paulo: Editora Santos; 2004. p. 109-28.

45. Unell L, Soderfeldt B, Halling A, Birkhed D. Explanatory models for clinically determined and symptom-reported caries indicators in an adult population. Acta Odontol Scand 1999; 57:132-8.
46. Riley III JL, Tomar SL, Gilbert GH. Smoking and smokeless tobacco: increased risk for oral pain. J Pain 2004; 5:218-25.

47. Barreto SM, Giatti L, Casado L, Moura L, Crespo C, Malta DC. Exposição ao tabagismo entre escolares no Brasil. Ciênc Saúde Coletiva 2010; 15:3027-34.

48. Leatherdale ST, Hammond D, Ahmed R. Alcohol, marijuana, and tobacco use patterns among youth in Canada. Cancer Causes Control 2008; 19:361-9.

49. Riley III JL, Gilbert GH, Heft MW. Orofacial pain: racial and sex differences among older adults. J Public Health Dent 2002; 62:132-9.

50. Brennan PL, Schutte KK, Moos RH. Pain and use of alcohol to manage pain: prevalence and 3-year outcomes among older problem and non-problem drinkers. Addiction 2005; 100:777-86.

51. Riley JL, King C. Self-report of alcohol use for pain in a multi-ethnic community sample. J Pain 2009; 10:944-52.

52. Roncalli AG. Epidemiologia das desigualdades em saúde bucal com foco em famílias. In: Moysés ST, Kriger L, Moysés SJ, organizadores. Saúde bucal das famílias: trabalhando com evidências. São Paulo: Editora Artes Médicas; 2008. p. 170-94.

53. Departamento de Atenção Básica, Secretaria de Atenção à Saúde, Ministério da Saúde. Cadernos de atenção básica no. 24: saúde na escola. Brasília: Ministério da Saúde; 2009. (Série B. Textos Básicos de Saúde).

54. Sheiham A, Watt RG. The common risk factor approach: a rational basis for promoting oral health. Community Dent Oral Epidemiol 2000; 28:399-406.

Recebido em 29/Dez/2010

Versão final reapresentada em 25/Jun/2011 Aprovado em 06/Jul/2011 